\title{
Intracavity Faraday Modulation Spectroscopy (INFAMOS): a tool for radical detection Supplementary Material
}

\author{
Michele Gianella, Tomas H.P. Pinto, Xia Wu, Grant A.D. Ritchie ${ }^{1}$ \\ Department of Chemistry, Physical and Theoretical Chemistry Laboratory, University of Oxford, South Parks Road, \\ Oxford OX1 3QZ, UK
}

\section{FARADAY ROTATION WITH STATIC MAGNETIC FIELD}

Consider an experiment consisting of a laser source, a first polarizer, a cell containing the sample with a longitudinal static magnetic field applied along its axis, a second polarizer (analyzer) and a photodetector (Fig. 1).

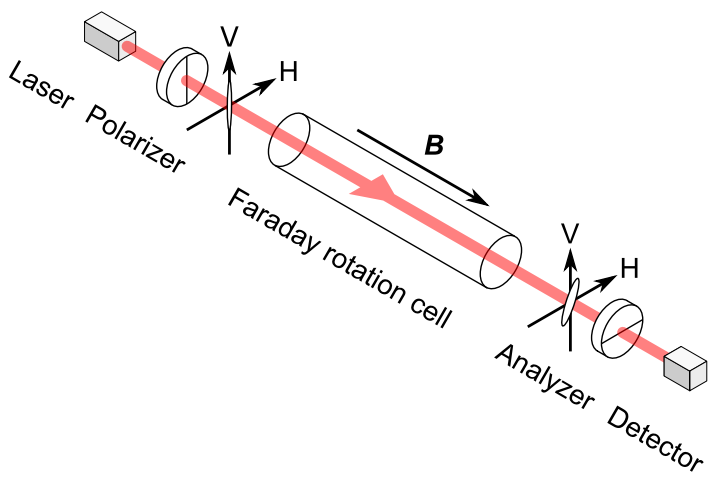

Figure 1. A simplified Faraday rotation experiment.

A plane wave traveling along the $z$-axis can be represented as a superposition of two orthogonal linearly polarized waves $(|\mathrm{V}\rangle$ : vertical polarization, $|\mathrm{H}\rangle$ : horizontal polarization), or as a superposition of two circularly polarized waves $(|\mathrm{L}\rangle$ : left-hand circular polarization (LHCP), $|\mathrm{L}\rangle=(|\mathrm{V}\rangle-\mathrm{i}|\mathrm{H}\rangle) / \sqrt{2} ;|\mathrm{R}\rangle$ : right-hand circular polarization (RHCP), $|\mathrm{R}\rangle=(|\mathrm{V}\rangle+\mathrm{i}|\mathrm{H}\rangle) / \sqrt{2})$. Ideally, the beam is linearly polarized after the first polarizer. However, to take into account the finite extinction ratio of the latter, we assume the wave to be elliptically polarized, with major semi-axis of length 1 along the vertical direction, minor semi-axis of length $\epsilon \ll 1$ along the horizontal direction, intensity $I=\left\langle\mathrm{E}_{\mathrm{in}} \mid \mathrm{E}_{\mathrm{in}}\right\rangle$ and frequency $\omega=k_{0} c$ :

$$
\begin{aligned}
\left|\mathrm{E}_{\mathrm{in}}\right\rangle & =\sqrt{I} e^{-\mathrm{i} \omega t}[|\mathrm{~V}\rangle+\mathrm{i} \epsilon|H\rangle] \\
& =\sqrt{\frac{I}{2}} e^{-\mathrm{i} \omega t}[(1-\epsilon)|\mathrm{L}\rangle+(1+\epsilon)|\mathrm{R}\rangle] .
\end{aligned}
$$

When the wave emerges from the paramagnetic sample after having propagated by a distance $L$, each circular component has experienced a phase-shift $\varphi^{\mathrm{L} / \mathrm{R}}=\phi^{\mathrm{L} / \mathrm{R}}+k_{0} L$, where $\phi^{\mathrm{L} / \mathrm{R}}$ is due to magnetic circular birefringence (MCB), and an attenuation $\delta^{\mathrm{L} / \mathrm{R}}$ due to absorption, which is different for LHCP and RHCP waves (magnetic circular dichroism, MCD). The single-pass transfer functions for each circular polarization state are

$$
h^{\mathrm{L} / \mathrm{R}}=e^{-\delta^{\mathrm{L} / \mathrm{R}}} e^{\mathrm{i} \varphi^{\mathrm{L} / \mathrm{R}}}=e^{\mathrm{i} k_{0} L} e^{-\bar{\delta}+\mathrm{i} \bar{\phi}} e^{\mp \mathscr{D} \pm \mathrm{i} \theta}=C e^{\mp \mathscr{D} \pm \mathrm{i} \theta},
$$

where we have introduced the average phase-shift $\bar{\phi}=\left(\phi^{\mathrm{L}}+\right.$ $\left.\phi^{\mathrm{R}}\right) / 2$, the average attenuation $\bar{\delta}=\left(\delta^{\mathrm{L}}+\delta^{\mathrm{R}}\right) / 2$, the Faraday rotation angle

$$
\theta=\frac{\phi^{\mathrm{L}}-\phi^{\mathrm{R}}}{2}
$$

the dichroism term

$$
\mathscr{D}=\frac{\delta^{\mathrm{L}}-\delta^{\mathrm{R}}}{2},
$$

and the common phase-shift and attenuation factor $C=$ $\exp \left(\mathrm{i} k_{0} L\right) \exp (-\bar{\delta}+\mathrm{i} \bar{\phi})$. If the magnetic field is limited to a region shorter than the cell length, a term $\alpha\left(L_{c}-L\right) / 2$ must be added to $\delta^{\mathrm{L} / \mathrm{R}}$ to account for the absorption that takes place outside of the field region ( $L_{c}$ : cell length, $L$ : field interaction length, $\alpha$ : absorption coefficient). This would affect the average attenuation $\bar{\delta}$ but not the dichroism $\mathscr{D}$. After the sample, the polarization state becomes:

$$
\begin{aligned}
\left|\mathrm{E}_{\text {out }}\right\rangle= & \sqrt{\frac{I}{2}} e^{-\mathrm{i} \omega t}\left[h^{\mathrm{L}}(1-\epsilon)|\mathrm{L}\rangle+h^{\mathrm{R}}(1+\epsilon)|\mathrm{R}\rangle\right] \\
= & C \sqrt{\frac{I}{2}} e^{-\mathrm{i} \omega t}\left[e^{-\mathscr{D}+\mathrm{i} \theta}(1-\epsilon)|\mathrm{L}\rangle\right. \\
& \left.+e^{+\mathscr{D}-\mathrm{i} \theta}(1+\epsilon)|\mathrm{R}\rangle\right],
\end{aligned}
$$

Neglecting dichroism $(\mathscr{D}=0)$, assuming $\epsilon=0$, and writing $|\mathrm{L}\rangle$ and $|\mathrm{R}\rangle$ as superpositions of linearly polarized waves $|\mathrm{V}\rangle$ and $|\mathrm{H}\rangle$, Eq. 5 simplifies to

$$
\left|\mathrm{E}_{\text {out }}\right\rangle=C \sqrt{I} e^{-\mathrm{i} \omega t}[\cos \theta|\mathrm{V}\rangle+\sin \theta|\mathrm{H}\rangle]
$$

which shows that MCB (without MCD) simply rotates the beam's polarization plane by $\theta$. Linearizing Eq. 5 for small values of $\theta$ and $\mathscr{D}$ yields

$$
\begin{aligned}
\left|\mathrm{E}_{\text {out }}\right\rangle \approx & C \sqrt{I} e^{-\mathrm{i} \omega t}[(1+\epsilon \mathscr{D}-\mathrm{i} \epsilon \theta)|\mathrm{V}\rangle \\
& +(\theta+\mathrm{i} \mathscr{D}+\mathrm{i} \epsilon)|\mathrm{H}\rangle]
\end{aligned}
$$

Recall that a superposition $c_{1} e^{\mathrm{i} \psi_{1}}|\mathrm{~V}\rangle+c_{2} e^{\mathrm{i} \psi_{2}}|\mathrm{H}\rangle$ with $c_{1}, c_{2} \in$ $\mathbb{R}$ is:

1. a linearly polarized wave if $\psi_{1}=\psi_{2}$;

2. a circularly polarized wave if $\psi_{1}-\psi_{2}=\pi / 2$ and $c_{1}=c_{2}$;

3. an elliptically polarized wave otherwise. 
Hence, the wave in Eq. 7 is elliptically polarized if $\mathscr{D} \neq 0$ or $\epsilon \neq 0$ (or both).

The analyzer acts on $\left|\mathrm{E}_{\text {out }}\right\rangle$ to produce the field at the detector $\left|\mathrm{E}_{\text {det }}\right\rangle=\mathscr{A}\left|\mathrm{E}_{\text {out }}\right\rangle$. The eigenstates of $\mathscr{A}$ are linearly polarized waves which are parallel and perpendicular, respectively, to the analyzer's axis:

$$
\mathscr{A}\left|\mathrm{A}_{\|}\right\rangle=\left|\mathrm{A}_{\|}\right\rangle, \quad \mathscr{A}\left|\mathrm{A}_{\perp}\right\rangle=\sqrt{\xi}\left|\mathrm{A}_{\perp}\right\rangle,
$$

where $\xi \ll 1$ is the (intensity) extinction ratio of the analyzer. The analyzer's axis is rotated by an angle $\beta$ with respect to the $|\mathrm{H}\rangle$ polarization. If $\beta=0$, then the analyzer is crossed with respect to the initial $|\mathrm{V}\rangle$ polarization, hence $\beta$ is called the uncrossing angle. The state $\left|\mathrm{A}_{\|}\right\rangle$is thus oriented at an angle of $\beta$ with respect to the $|\mathrm{H}\rangle$ state (or $90^{\circ}-\beta$ with respect to $|\mathrm{V}\rangle$ ), and $\left|\mathrm{A}_{\perp}\right\rangle$ is perpendicular to $\left|\mathrm{A}_{\|}\right\rangle$. By expressing $|\mathrm{L}\rangle,|\mathrm{R}\rangle$ in the $\left|\mathrm{A}_{\|}\right\rangle,\left|\mathrm{A}_{\perp}\right\rangle$ basis,

$$
\begin{aligned}
|\mathrm{L}, \mathrm{R}\rangle & =\frac{1}{\sqrt{2}}[|\mathrm{~V}\rangle \mp \mathrm{i}|\mathrm{H}\rangle], \\
& =\frac{1}{\sqrt{2}}\left[\left(\sin \beta\left|\mathrm{A}_{\|}\right\rangle+\cos \beta\left|\mathrm{A}_{\perp}\right\rangle\right)\right. \\
& \left.\mp \mathrm{i}\left(\cos \beta\left|\mathrm{A}_{\|}\right\rangle-\sin \beta\left|\mathrm{A}_{\perp}\right\rangle\right)\right] \\
& =\frac{e^{ \pm \mathrm{i} \beta}}{\sqrt{2}}\left[\left|\mathrm{~A}_{\perp}\right\rangle \mp \mathrm{i}\left|\mathrm{A}_{\|}\right\rangle\right],
\end{aligned}
$$

we can recast Eq. 5 into a form more suitable for the following calculations,

$$
\left|\mathrm{E}_{\text {out }}\right\rangle=C \frac{\sqrt{I}}{2} e^{-\mathrm{i} \omega t}\left[c_{\perp}\left|\mathrm{A}_{\perp}\right\rangle+c_{\|}\left|\mathrm{A}_{\|}\right\rangle\right],
$$

where

$$
\begin{aligned}
& c_{\perp}=e^{-\mathscr{D}+\mathrm{i} \theta} e^{\mathrm{i} \beta}(1-\epsilon)+e^{\mathscr{D}-i \theta} e^{-\mathrm{i} \beta}(1+\epsilon), \\
& c_{\|}=\mathrm{i}\left[e^{\mathscr{D}-\mathrm{i} \theta} e^{-\mathrm{i} \beta}(1+\epsilon)-e^{-\mathscr{D}+\mathrm{i} \theta} e^{\mathrm{i} \beta}(1-\epsilon)\right] .
\end{aligned}
$$

The intensity measured at the detector, $I_{\text {det }}=\left\langle\mathrm{E}_{\text {det }} \mid \mathrm{E}_{\operatorname{det}}\right\rangle$, is then:

$$
\begin{aligned}
I_{\mathrm{det}}= & \left\langle\mathrm{E}_{\mathrm{det}} \mid \mathrm{E}_{\mathrm{det}}\right\rangle \\
= & \left\langle\mathrm{E}_{\text {out }}\left|\mathscr{A}^{\dagger} \mathscr{A}\right| \mathrm{E}_{\text {out }}\right\rangle \\
= & \frac{I|C|^{2}}{4}\left[\left\langleA_{\perp}\left|c_{\perp}^{*}+\left\langle A_{\|}\right| c_{\|}^{*}\right] \mathscr{A}^{\dagger} \mathscr{A}\left[c_{\perp}\left|\mathrm{A}_{\perp}\right\rangle+c_{\|}\left|\mathrm{A}_{\|}\right\rangle\right]\right.\right. \\
= & \frac{I|C|^{2}}{4}\left[\xi\left|c_{\perp}\right|^{2}+\left|c_{\|}\right|^{2}\right] \\
= & \frac{I e^{-2 \bar{\delta}}}{2}[\cosh (2 \mathscr{D})-\cos (2 \theta+2 \beta) \\
& +\left(\xi+\epsilon^{2}+\epsilon^{2} \xi\right) \cosh (2 \mathscr{D}) \\
& +\left(\xi+\epsilon^{2}-\epsilon^{2} \xi\right) \cos (2 \theta+2 \beta) \\
& +2(1+\xi) \epsilon \sinh (2 \mathscr{D})]
\end{aligned}
$$

Between Eq. 15 and 16 we have used Eq. 8 and the orthonormality relationships for $\left|A_{\|}\right\rangle,\left|A_{\perp}\right\rangle$. In Eq. 16 we have inserted
Eqs. 11 and 12 and obtained a rather cumbersome expression for $I_{\text {det }}$, similar to the one obtained by Westberg. ${ }^{1}$ The first term (17) describes the measured signal if an ideal linearly polarized beam $(\epsilon=0)$ and an ideal analyzer $(\xi=0)$ had been used. It includes both MCB and MCD. It is the dominant term, and we can think of the following terms as corrections that take into account the initial polarization of the beam not being perfectly linear $(\epsilon \neq 0)$ and the analyzer having a finite extinction ratio $(\xi \neq 0)$.

We can simplify this result significantly if we assume weak $\operatorname{MCD}(\mathscr{D} \ll 1)$ and MCB $(\theta \ll 1)$, weak absorption $(\bar{\delta} \ll 1)$, a good analyzer $(\xi \ll 1)$, and $\epsilon \ll 1$. By linearizing the various summands and the $e^{-2 \bar{\delta}}$ factor and only retaining the leading terms we obtain:

$$
\begin{aligned}
I_{\mathrm{det}} \approx & \frac{I}{2}[\underbrace{1+\xi-(1-\xi) \cos (2 \beta)+\epsilon^{2}}_{\text {wavelength-independent }} \\
& +\underbrace{2 \bar{\delta}[\cos (2 \beta)-1]}_{\text {absorption }}+\underbrace{2 \theta \sin 2 \beta}_{\mathrm{MCB}}+\underbrace{4 \epsilon \mathscr{D}+2 \mathscr{D}^{2}}_{\mathrm{MCD}}] .
\end{aligned}
$$

Notice that while the leading term with respect to MCB is $\sim \theta$, for MCD there is no term of order $\mathscr{D}$ : the two lowest order terms involving dichroism are $\sim \mathscr{D}^{2}$ and $\sim \epsilon \mathscr{D}$. This justifies the often made assumption that the effect of MCD is negligible compared to MCB. Notice also, however, that while the signal arising from MCB is dependent on the uncrossing angle $\beta$ (and can therefore be very small if $\beta \approx 0$ ), the signal caused by MCD does not depend on $\beta$. In particular, in the crossed position $(\beta=0)$ dichroism will dominate over birefringence. We can further simplify Eq. 21 by assuming a small uncrossing angle, $\beta \ll 1$, and only retaining terms up to $\sim \beta^{2}$ :

$$
\begin{aligned}
I_{\text {det }}= & {[\underbrace{\underbrace{\xi+\beta^{2}+\epsilon^{2} / 2}}_{\text {wavelength-independent }}-\underbrace{2 \beta^{2} \bar{\delta}}_{\text {absorption }}} \\
& +\underbrace{2 \beta \theta}_{\mathrm{MCB}}+\underbrace{2 \epsilon \mathscr{D}+\mathscr{D}^{2}}_{\mathrm{MCD}}] .
\end{aligned}
$$

The final result (Eq. 22) shows that, in the crossed position $(\beta=0)$, intensity is transmitted through the analyzer for three reasons:

1. $\xi$-term: the analyzer extinction ratio $\xi>0$ lets through a fraction $\xi$ of the incident $|\mathrm{V}\rangle$ component;

2. $\epsilon^{2} / 2$-term: if the beam is initially elliptically polarized, a fraction thereof will be transmitted through the analyzer;

3. $2 \epsilon \mathscr{D}$ and $\mathscr{D}^{2}$-term: MCD increases the "ellipticity" of the beam's polarization further; 
By uncrossing the analyzer, the intensity on the detector changes for three reasons:

1. $\beta^{2}$-term: the wave incident on the analyzer now has a polarization component parallel to the analyzer's axis, which is transmitted unattenuated;

2. $2 \beta \theta$-term: MCB rotates the beam's polarization axis, increasing the component of the polarization parallel to the analyzer's axis;

3. $-2 \beta^{2} \bar{\delta}$-term: the "normal" (non-MCD) absorption $2 \bar{\delta}$, attenuated by the analyzer by $\beta^{2}$.

The electronic signal output by the detector is

$$
\begin{aligned}
\mathcal{S} & =\mathcal{G} I_{\mathrm{det}} \\
& =\mathcal{G} I\left[\xi+\beta^{2}+\epsilon^{2} / 2-2 \beta^{2} \bar{\delta}+2 \beta \theta+2 \epsilon \mathscr{D}+\mathscr{D}^{2}\right] \\
& =\mathcal{S}_{0}+\delta \mathcal{S}
\end{aligned}
$$

where $\mathcal{G}$ is an instrumental factor that takes detector responsivity and amplifier gain into account, $\mathcal{S}_{0}=\mathcal{G} I\left[\xi+\beta^{2}+\epsilon^{2} / 2\right]$ is the wavelength-independent part of the signal, and $\delta \mathcal{S}=$ $\mathcal{G} I\left[-2 \beta^{2} \bar{\delta}+2 \beta \theta+2 \epsilon \mathscr{D}+\mathscr{D}^{2}\right]$ is the signal variation induced by absorption, MCB, and MCD.

\section{FARADAY ROTATION WITH SINUSOIDALLY VARYING MAGNETIC FIELD}

With a sinusoidally varying magnetic field, $B(t)=$ $B_{0} \cos (\Omega t)$, the intensity measured at the detector $I_{\text {det }}$ (Eq. 22) becomes time-dependent. A lock-in amplifier mixes (multiplies) the input signal with a cosine reference at the frequency $\Omega$ and applies a low-pass filter (time-average) to it. Since the Faraday rotation angle $\theta$ and the dichroism term $\mathscr{D}$ in Eq. 22 are now periodic (with period $2 \pi / \Omega$ ), they can be written as Fourier series:

$$
\begin{aligned}
\theta(t) & =\sum_{k=-\infty}^{\infty} \Theta_{k} e^{\mathrm{i} k \Omega t} \\
\mathscr{D}(t) & =\sum_{k=-\infty}^{\infty} \mathfrak{D}_{k} e^{\mathrm{i} k \Omega t} \\
\mathscr{D}^{2}(t) & =\sum_{k=-\infty}^{\infty} \mathfrak{D}_{k}^{(2)} e^{\mathrm{i} k \Omega t} \\
\cos (\Omega t) & =\frac{1}{2}\left(e^{\mathrm{i} \Omega t}+e^{-\mathrm{i} \Omega t}\right)
\end{aligned}
$$

where $\Theta_{k}, \mathfrak{D}_{k}$ and $\mathfrak{D}_{k}^{(2)}$ are the (complex) Fourier coefficients of $\theta, \mathcal{D}$ and $\mathcal{D}^{2}$, respectively. The mean absorption $\bar{\delta}$ is periodic as well, but its period is half that of the magnetic field. ${ }^{2}$ Thus, its Fourier representation only contains even multiples of $\Omega$ $(0, \pm 2 \Omega, \pm 4 \Omega \ldots)$. As we are only interested in the first harmonic $(\Omega), \bar{\delta}$ gives no contribution to the lock-in output. The Fourier coefficients of $\mathscr{D}^{2}$ can be derived from those of $\mathscr{D}$ via the convolution theorem, $\mathfrak{D}_{k}^{(2)}=\sum_{k^{\prime}} \mathfrak{D}_{k^{\prime}} \mathfrak{D}_{k-k^{\prime}}$. Since both $\theta$ and $\mathscr{D}$ are real-valued, we have $\Theta_{k}=\Theta_{-k}^{*}$ and $\mathfrak{D}_{k}=\mathfrak{D}_{-k}^{*}$.
The spectrum of the lock-in input signal (Eq. 23) without the constant and absorption terms,

$$
\delta \mathcal{S}(t)=\mathcal{G} I\left[2 \beta \theta(t)+2 \epsilon \mathscr{D}(t)+\mathscr{D}^{2}(t)\right],
$$

therefore consists of oscillating components with discrete frequencies $k \Omega(k \in \mathbb{Z})$, called the harmonics of the signal. The multiplication with the cosine produces, for each harmonic $k \Omega$, a sum-frequency $((k+1) \Omega)$ and a difference-frequency $((k-1) \Omega)$ term:

$$
\begin{aligned}
\delta \mathcal{S}(t) \cos (\Omega t)= & \frac{\mathcal{G} I}{2} \sum_{k=-\infty}^{\infty}\left(2 \beta \Theta_{k}+2 \epsilon \mathfrak{D}_{k}+\mathfrak{D}_{k}^{(2)}\right) \\
& \times\left(e^{\mathrm{i}(k+1) \Omega t}+e^{\mathrm{i}(k-1) \Omega t}\right) .
\end{aligned}
$$

For the first harmonic $(k= \pm 1)$ of $\mathcal{S}$, the sum- and differencefrequency terms on the right-hand side of Eq. 29 are

$$
\begin{array}{r}
\left(2 \beta \mathfrak{R}\left[\Theta_{1}\right]+2 \epsilon \mathfrak{R}\left[\mathfrak{D}_{1}\right]+\mathfrak{R}\left[\mathfrak{D}_{1}^{(2)}\right]\right) \\
+\left(2 \beta \mathfrak{K}\left[\Theta_{1} e^{\mathrm{i} 2 \Omega t}\right]+2 \epsilon \mathfrak{K}\left[\mathfrak{D}_{1} e^{\mathrm{i} 2 \Omega t}\right]+\mathfrak{R}\left[\mathfrak{D}_{1}^{(2)} e^{\mathrm{i} 2 \Omega t}\right]\right),
\end{array}
$$

i.e. the difference-frequency terms are constant $(\Omega-\Omega=0)$ and the sum-frequency terms oscillate with frequency $\Omega+\Omega=$ $2 \Omega$. For all higher harmonics, $|k| \geq 2$, all terms oscillate (with frequencies $(k \pm 1) \Omega)$. By low-pass filtering the right-hand side of Eq. 29, only the constant terms arising from the first harmonic (Eq. 30) survive. The oscillating components can be suppressed by choosing the time constant of the low-pass filter appropriately large. The output of the lock-in amplifier is then simply given by

$$
X=\mathcal{G} I\left(\beta \mathfrak{R}\left[\Theta_{1}\right]+\epsilon \mathfrak{R}\left[\mathfrak{D}_{1}\right]+\mathfrak{R}\left[\mathfrak{D}_{1}^{(2)}\right] / 2\right) .
$$

Depending on the initial polarization state of the beam, one of the two dichroism terms may be negligible compared to the other. A possible factor of $\sqrt{2}$, arising from whether we employ amplitudes or r.m.s. amplitudes, can be absorbed into $\mathcal{G}$ if necessary. The Fourier coefficient $\mathfrak{R}\left[\Theta_{1}\right]$ can be expressed as a function of the first Fourier coefficient of the dispersive lineshape function $\chi\left(\mathscr{W}_{M}(t), \mathscr{V}\right)$ (see Section IV below), where $\mathscr{W}_{M}(t)$ varies sinusoidally, making $\chi$ periodic. $^{3}$

\section{FARADAY ROTATION WITH CAVITY-ENHANCEMENT}

Consider a linear optical cavity of length $L$ made of two highly reflective mirrors (reflectivity $R=1-T \approx 1$ ). The transfer functions of such a system for circularly polarized light are: ${ }^{4}$

$$
\begin{aligned}
h_{\mathrm{cav}}^{\mathrm{L} / \mathrm{R}} & =\frac{T \exp \left(-\delta^{\mathrm{L} / \mathrm{R}}+\mathrm{i} \varphi^{\mathrm{L} / \mathrm{R}}\right)}{1-R \exp \left(-2 \delta^{\mathrm{L} / \mathrm{R}}+2 \mathrm{i} \varphi^{\mathrm{L} / \mathrm{R}}\right)} \\
& =\frac{C T \exp (\mp \mathscr{D} \pm \mathrm{i} \theta)}{1-R C^{2} \exp (\mp 2 \mathscr{D} \pm 2 \mathrm{i} \theta)},
\end{aligned}
$$

where we have used the same symbols as in Eq. 2. Strictly speaking, the resonance frequencies are different for LHCP and 
RHCP waves due to the different phase-shifts they experience while circulating within the cavity. We can, however, assume that the contribution to the phase-shift arising from MCB is much smaller than the "plane wave" term, $\phi^{\mathrm{L} / \mathrm{R}} \ll k L$, so that the resonances of $h_{\mathrm{cav}}^{\mathrm{L} / \mathrm{R}}$ are essentially still located at $k_{q}=$ $q \pi / L, q \in \mathbb{N}\left(\right.$ or $\left.v_{q}=q c / 2 L\right)$. In the limit of weak absorption, the factor $C$ further simplifies to $C \approx \pm \exp (-\bar{\delta}) \approx \pm 1$. We can write $h_{\text {cav }}^{\mathrm{L} / \mathrm{R}}$ as a Taylor expansion for small values of $\theta$ and $\mathscr{D}$ at the $q$ th resonance frequency:

$$
\begin{aligned}
& h_{\mathrm{cav}, q}^{\mathrm{L}} \approx \pm\left[1+\frac{2 R}{1-R}(-\mathscr{D}+\mathrm{i} \theta)\right], \\
& h_{\mathrm{cav}, q}^{\mathrm{R}} \approx \pm\left[1+\frac{2 R}{1-R}(+\mathscr{D}-\mathrm{i} \theta)\right] .
\end{aligned}
$$

The factor $2 R(1-R)^{-1}$ is the cavity enhancement factor and can be expressed through the finesse $\mathcal{F}$ as $2 R(1-R)^{-1} \approx 2 \mathcal{F} / \pi$. Finally, by replacing the single-pass transfer functions $h^{\mathrm{L} / \mathrm{R}}$ in Eq. 5 with $h_{\text {cav }, q}^{\mathrm{L} / \mathrm{R}}$, we obtain at the output of the cavity

$$
\begin{aligned}
\left|\mathrm{E}_{\mathrm{cav}}\right\rangle= & \sqrt{\frac{I}{2}} e^{-\mathrm{i} \omega t}\left(h_{\mathrm{cav}, q}^{\mathrm{L}}(1-\epsilon)|\mathrm{L}\rangle+h_{\mathrm{cav}, q}^{\mathrm{R}}(1+\epsilon)|\mathrm{R}\rangle\right) \\
\approx & \pm \sqrt{I} e^{-\mathrm{i} \omega t}\left[\left(1+\epsilon \frac{2 \mathcal{F}}{\pi} \mathscr{D}-\mathrm{i} \epsilon \frac{2 \mathcal{F}}{\pi} \theta\right)|\mathrm{V}\rangle\right. \\
& \left.+\left(\frac{2 \mathcal{F}}{\pi} \theta+\mathrm{i} \frac{2 \mathcal{F}}{\pi} \mathscr{D}+\mathrm{i} \epsilon\right)|\mathrm{H}\rangle\right] \\
\approx & \pm \sqrt{I} e^{-\mathrm{i} \omega t}\left[\left(1+\epsilon \mathscr{D} \text { cav }-\mathrm{i} \epsilon \theta_{\mathrm{cav}}\right)|\mathrm{V}\rangle\right. \\
& \left.+\left(\theta_{\mathrm{cav}}+\mathrm{i} \mathscr{D}_{\mathrm{cav}}+\mathrm{i} \epsilon\right)|\mathrm{H}\rangle\right] .
\end{aligned}
$$

Comparison of Eq. 36 with 7 shows that the effect of the cavity is to multiply the single-pass Faraday rotation angle $\theta$ and dichroism $\mathscr{D}$ by the cavity enhancement factor $2 \mathcal{F} / \pi$,

$$
\begin{aligned}
\theta_{\text {cav }} & =\frac{2 \mathcal{F}}{\pi} \theta, \\
\mathscr{D}_{\text {cav }} & =\frac{2 \mathcal{F}}{\pi} \mathscr{D} .
\end{aligned}
$$

Notice that $\epsilon$ is not enhanced by the cavity, since it is a property of the initial polarization state of the beam. We note that linear birefringence and polarization-dependent losses ${ }^{5,6}$ of the cavity mirrors are not accounted for in this simple model.

\section{PHASE-SHIFT INDUCED BY ZEEMAN-SPLIT TRANSITION ON CIRCULARLY POLARIZED LIGHT}

\section{Definitions}

- $\phi_{M}^{+}\left(\delta_{M}^{+}\right)$: phase-shift (attenuation) experienced by a LHCP wave due to the Zeeman-split transition $M \rightarrow$ $M+1$

- $\phi_{M}^{-}\left(\delta_{M}^{-}\right)$: phase-shift (attenuation) experienced by a RHCP wave due to the Zeeman-split transition $M \rightarrow$ $M-1$
- $\phi^{\mathrm{L}}\left(\delta^{\mathrm{L}}\right)$ : total phase-shift (attenuation) experienced by a LHCP wave

- $\phi^{\mathrm{R}}\left(\delta^{\mathrm{R}}\right)$ : total phase-shift (attenuation) experienced by a RHCP wave

- $S$ : line strength (integrated absorption cross-section) $\left[\mathrm{cm}^{2} \mathrm{~cm}^{-1} /\right.$ molecule $]$

- $N$ : absorber number density $\left[\mathrm{cm}^{-3}\right]$

- $L$ : absorption and interaction with magnetic field path length, assumed to be identical [cm]

- $\bar{S}_{M}^{+}\left(\bar{S}_{M}^{-}\right)$: relative line strength of the Zeeman-split transition $M \rightarrow M+1(M \rightarrow M-1)$ [unitless]

- $\chi, \eta$ : lineshape functions for dispersion and absorption, respectively [unitless]

- $w(x+\mathrm{i} y)=w(z)=e^{-z^{2}} \operatorname{erfc}(-\mathrm{i} z)$ : scaled complementary error function [unitless]

- $\mathscr{W}_{M}^{+}\left(\mathscr{W}_{M}^{-}\right)$: Doppler-width normalized detuning of the laser frequency from the Zeeman-shifted transition $M \rightarrow M+1(M \rightarrow M-1)$ [unitless]

- $\mathscr{V}$ : Voigt parameter [unitless]

- $J\left(J^{\prime}\right)$ : lower (upper) state angular momentum quantum number [unitless]

- $g\left(g^{\prime}\right)$ : g-factor of the lower (upper) state [unitless]

- $M$ : lower state magnetic quantum number [unitless]

- $\gamma_{\mathrm{G}}\left(\gamma_{\mathrm{L}}\right)$ : Gaussian (Lorenztian) HWHM $\left[\mathrm{cm}^{-1}\right]$

When a plane wave propagates through a medium, the molecules (or atoms) act as resonant antennas and re-emit the incident field. The resonances correspond to allowed transitions between the molecules' energy eigenstates. As the frequency of the incident wave approaches one of the allowed transitions, the amplitude and phase of the re-emitted wave start to change significantly, analogous to the motion of a classical oscillator being driven close to resonance. This results in an attenuation and phase-shift of the plane wave that can be written in the form

$$
E(z, t)=E_{0} e^{-\mathrm{i}\left(\omega t-k_{0} z\right)} e^{i k(\omega) z}
$$

where $k(\omega)$ is the complex wavenumber due to the transition(s), and $k_{0}=\omega / c$. The phase-shift and attenuation induced by the transition(s) after propagation over a distance $L$ are then

$$
\begin{aligned}
& \phi=\mathfrak{R}[k] L \\
& \delta=\mathfrak{J}[k] L .
\end{aligned}
$$

In Ref. 3, Westberg et al have derived expressions for the real and imaginary part of $k$ using the line strength of the transition, which is conveniently tabulated in the HITRAN database, ${ }^{7}$ and the scaled complementary error function (Faddeeva function). 
Here, we consider fundamental rovibrational transitions of NO $\left(1800-1950 \mathrm{~cm}^{-1}\right)$. The states involved adhere to Hund's coupling case (a), in which the total angular momentum $J$ is a good quantum number (at least at low $J$ ). The lower (upper) state of the transition is $2 J+1-\left(2 J^{\prime}+1\right)$-fold degenerate. The application of a magnetic field lifts this degeneracy and produces $2 J+1\left(2 J^{\prime}+1\right)$ energy and $J_{z^{-}}$-eigenstates with quantum numbers $M=-J,-J+1, \ldots, J\left(M^{\prime}=-J^{\prime},-J^{\prime}+1, \ldots, J^{\prime}\right)$. If the incident wave propagates parallel to the magnetic field, left-hand circularly polarized (LHCP) waves interact only with $M \rightarrow M+1$ sub-transitions, and right-hand circularly polarized (RHCP) waves only with $M \rightarrow M-1$. The transitions $M \rightarrow M$ are forbidden in this configuration.

The phase-shift and attenuation of a LHCP wave due to the $M \rightarrow M+1$ sub-transition are given by:

$$
\phi_{M}^{+}=\frac{S N L}{2} \bar{S}_{M}^{+} \chi\left(\mathscr{W}_{M}^{+}, \mathscr{V}\right)
$$

and

$$
\delta_{M}^{+}=\frac{S N L}{2} \bar{S}_{M}^{+} \eta\left(\mathscr{W}_{M}^{-}, \mathscr{V}\right)
$$

$\bar{S}_{M}^{+}$, the intensity of the transition $M \rightarrow M+1$ relative to the total intensity $S$, can be expressed through the Wigner $3-j$ symbol $^{8}$

$$
\bar{S}_{M}^{+}=3\left(\begin{array}{ccc}
J^{\prime} & 1 & J \\
-(M+1) & 1 & M
\end{array}\right)^{2}
$$

The dispersive and absorptive lineshape functions $\chi$ and $\eta$ are given by the imaginary and real part, respectively, of the scaled complementary error function $w$ (Ref. 9, Ch. 7):

$$
\begin{aligned}
& \chi\left(\mathscr{W}_{M}^{+}, \mathscr{V}\right)=-\frac{\sqrt{\ln 2}}{\gamma_{\mathrm{G}}} \sqrt{\frac{1}{\pi}} \mathfrak{J}\left[w\left(\mathscr{W}_{M}^{+}+\mathrm{i} \mathscr{V}\right)\right] \\
& \eta\left(\mathscr{W}_{M}^{+}, \mathscr{V}\right)=+\frac{\sqrt{\ln 2}}{\gamma_{\mathrm{G}}} \sqrt{\frac{1}{\pi}} \mathfrak{R}\left[w\left(\mathscr{W}_{M}^{+}+\mathrm{i} \mathscr{V}\right)\right] .
\end{aligned}
$$

The Doppler-width normalized detuning of the laser frequency $v$ from the centre of the Zeeman-shifted transition $\mathscr{W}_{M}^{+}$is

$$
\mathscr{W}_{M}^{+}=\frac{\sqrt{\ln 2}}{\gamma_{\mathrm{G}}}\left\{v-\left[v_{0}-\left((M+1) g^{\prime}-M g\right) \mu_{B} B\right]\right\},
$$

where $v_{0}$ is the centre of the transition in the zero-field case and $\gamma_{\mathrm{G}}=v_{0} \sqrt{2 k T \ln 2 / m} / c$ is the Doppler width (HWHM). The Doppler-width normalized Lorentzian width (also called the Voigt parameter) is

$$
\mathscr{V}=\left(\sqrt{\ln 2} / \gamma_{\mathrm{G}}\right) \gamma_{\mathrm{L}}
$$

At low concentrations, as is the case in this article, selfbroadening can be neglected and the Lorentzian width can be expressed through the air-broadening parameter and the total gas pressure.

For a RHCP, which interacts only with transitions $M \rightarrow$ $M-1$, substitute the + superscript with - and $M+1$ with
$M-1$ in Eqs. 42, 43, 45, 46, and 47, and change the relative intensity to

$$
\bar{S}_{M}^{-}=3\left(\begin{array}{ccc}
J^{\prime} & 1 & J \\
-(M-1) & -1 & M
\end{array}\right)^{2}
$$

Notice the change of sign in two places in the second row. The total phase-shift and attenuation experienced by a LHCP (RHCP) wave is the sum of Eq. 42 and 43 over all $M$ values:

$$
\begin{aligned}
\phi^{\mathrm{L}} & =\sum_{M=-J}^{J} \phi_{M}^{+}=\frac{S N L}{2} \sum_{M=-J}^{J} \bar{S}_{M}^{+} \chi\left(\mathscr{W}_{M}^{+}, \mathscr{V}\right) \\
\phi^{\mathrm{R}} & =\sum_{M=-J}^{J} \phi_{M}^{-}=\frac{S N L}{2} \sum_{M=-J}^{J} \bar{S}_{M}^{-} \chi\left(\mathscr{W}_{M}^{-}, \mathscr{V}\right) \\
\delta^{\mathrm{L}} & =\sum_{M=-J}^{J} \delta_{M}^{+}=\frac{S N L}{2} \sum_{M=-J}^{J} \bar{S}_{M}^{+} \eta\left(\mathscr{W}_{M}^{+}, \mathscr{V}\right) \\
\delta^{\mathrm{R}} & =\sum_{M=-J}^{J} \delta_{M}^{-}=\frac{S N L}{2} \sum_{M=-J}^{J} \bar{S}_{M}^{-} \eta\left(\mathscr{W}_{M}^{-}, \mathscr{V}\right) .
\end{aligned}
$$

\section{MEASUREMENT BANDWIDTH}

The measurement bandwidth of an INFAMOS spectrum is given by three different types of averaging: (i) lock-in amplifier low-pass filtering; (ii) averaging of multiple data points acquired on a single cavity resonance (with gating); (iii) averaging across successively acquired spectra. Here, we will derive the combined equivalent bandwidth.

(i) The lock-in time constant was set to $t_{c}=100 \mu \mathrm{s}$ with a $24 \mathrm{~dB} /$ oct roll-off, corresponding to an equivalent noise bandwidth of $780 \mathrm{~Hz} .{ }^{10}$ The filter transfer function $\mathscr{F}_{1}$ for the power spectral density (PSD) of the signal is given by

$$
\mathscr{F}_{1}(f)=\left|\frac{1}{\left(1+2 \pi \mathrm{i} f t_{c}\right)^{4}}\right|^{2}
$$

and is plotted in Fig. 2.

(ii) The lock-in output signal is sampled by the DAQ board every $\delta t=10 \mu \mathrm{s}$ (sampling rate $\left.f_{s}=(\delta t)^{-1}=100 \mathrm{kSa} / \mathrm{s}\right)$. The assumption that averaging reduces the bandwidth by a factor of $N$ (number of points averaged) is only true if the individual data points being averaged are statistically independent. Sampling a $780-\mathrm{Hz}$ bandwidth signal at $100 \mathrm{kSa} / \mathrm{s}$ does not provide statistically independent points. ${ }^{11}$ If we indicate the lock-in output signal with $X_{k}$, then the moving average $\bar{X}_{k}$ over the previous $N_{\mathrm{g}}$ points is given by

$$
\bar{X}_{k}=\frac{1}{N_{\mathrm{g}}} \sum_{j=0}^{N_{\mathrm{g}}-1} X_{k-j}
$$

From the spectrum of $X$,

$$
X(f)=\sum_{k=-\infty}^{\infty} X_{k} e^{2 \pi \mathrm{i} f k \delta t}
$$




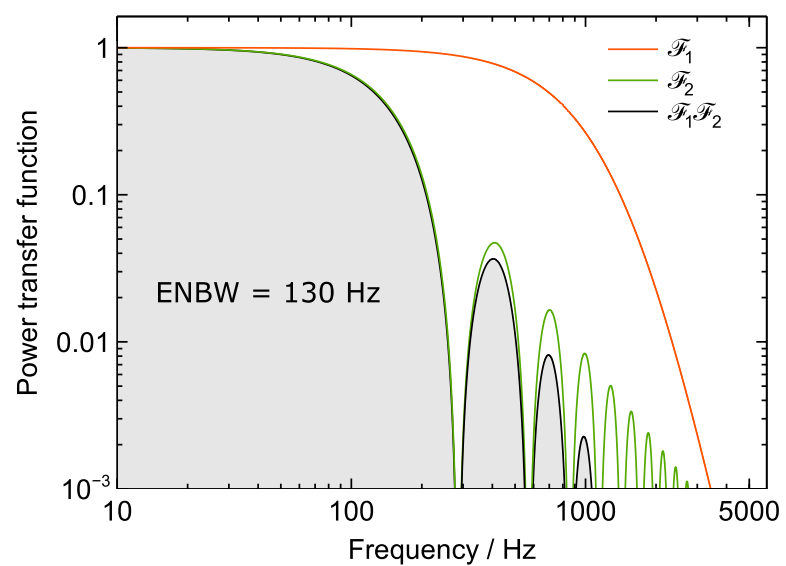

Figure 2. Filter transfer functions for the lock-in low-pass filter $\left(\mathscr{F}_{1}\right)$, the moving average filter over 360 points $\left(\mathscr{F}_{2}\right)$, and the combined function $\left(\mathscr{F}_{1} \mathscr{F}_{2}\right)$.

and with Eq. 55 we can derive the spectrum of $\bar{X}$ and, by taking its magnitude squared, the PSD of $\bar{X}$ :

$$
|\bar{X}(f)|^{2}=\frac{1}{N_{\mathrm{g}}^{2}}\left|\frac{1-e^{2 \pi i N_{\mathrm{g}} f \delta t}}{1-e^{2 \pi \mathrm{i} f \delta t}}\right|^{2}|\mathcal{X}(f)|^{2} .
$$

The factor before $|X(f)|^{2}$,

$$
\mathscr{F}_{2}=\frac{1}{N_{\mathrm{g}}^{2}}\left|\frac{1-e^{2 \pi i N_{\mathrm{g}} f \delta t}}{1-e^{2 \pi \mathrm{i} f \delta t}}\right|^{2},
$$

is the transfer function of the moving average filter, and is shown in Fig. 2 for $N_{\mathrm{g}}=360$ (typical number of points available at each cavity resonance after gating, corresponding to $3.5 \mathrm{~ms}$ of data). The combined transfer function $\mathscr{F}_{1} \mathscr{F}_{2}$ is also given in Fig. 2. Its equivalent noise bandwidth (ENBW) was numerically computed to be $130 \mathrm{~Hz}$.

(iii) Finally, we must consider the averaging across different spectra. This can again be considered a moving average, but with the data points spaced in time by the scan duration $(0.5 \mathrm{~s})$. In this case, the individual data points are separated enough that they can be considered statistically independent, and averaging $N$ spectra reduces the bandwidth by a factor $N$. We then obtain the overall measurement bandwidth:

$$
\mathscr{B}=\frac{130 \mathrm{~Hz}}{N},
$$

and in particular for 170 averaged spectra, $\mathscr{B}=0.77 \mathrm{~Hz}$.

\section{REFERENCES}

${ }^{1}$ J. Westberg, Faraday Modulation Spectroscopy, Ph.D. thesis, Umea University (2013).

${ }^{2}$ As a function of magnetic field, the attenuation factors obey the following relationship: $\delta^{\mathrm{L}}(-B)=\delta^{\mathrm{R}}(+B)$. This can be shown in the following way: from Eq. 47 it's easy tho show that $\mathscr{W}_{M}^{+}(B)=\mathscr{W}_{-M}^{-}(-B)$, meaning that the centre frequency of the (LHCP) sub-transition $M \rightarrow M+1$ when the field strength is $B$ is the same as for the (RHCP) sub-transition $-M \rightarrow-M-1$ when the field strength is $-B$. Assume that Figs. 1d and 1e (in the article) are plotted for a field strength of $B$. To plot them for a field strength of $-B$ one then simply has to mirror the existing plots around the $v=v_{0}$ vertical line. Since the mean attenuation $\bar{\delta}$ is an odd function (Fig. 1e in the article), we have $\bar{\delta}(-B)=\bar{\delta}(B)$. Since changing the sign of $B$ is equivalent to a time-shift $t \rightarrow t+T / 2, B(t+T / 2)=-B(t)$, for $\bar{\delta}$ we have $\bar{\delta}(B(t+T / 2))=\bar{\delta}(-B(t))=\bar{\delta}(B(t))$, i.e. $\bar{\delta}$ is $T / 2$-periodic.

${ }^{3}$ J. Westberg, L. Lathdavong, C. M. Dion, J. Shao, P. Kluczynski, S. Lundqvist, and O. Axner, Journal of Quantitative Spectroscopy and Radiative Transfer 111, 2415 (2010).

${ }^{4}$ Circular polarizations merely experience a phase-shift and attenuation which can be represented by multiplication by a complex phase factor (the transfer function). Linear polarizations, on the other hand, experience a rotation of the polarization plane. The transfer function for a linearly polarized wave is not overly complicated (see for example Ref. 12), but requires the use of Jones matrices.

${ }^{5}$ D. Jacob, M. Oger, M. Vallet, F. Bretenaker, and A. L. Floch, Opt. Lett. 20, 671 (1995).

${ }^{6}$ H. Huang and K. K. Lehmann, Applied Optics 47, 3817 (2008).

${ }^{7}$ L. S. Rothman, I. E. Gordon, Y. Babikov, A. Barbe, D. C. Benner, P. F. Bernath, M. Birk, L. Bizzocchi, V. Boudon, L. R. Brown, A. Campargue, K. Chance, L. H. Coudert, V. M. Devi, B. J. Drouin, A. Fayt, J.-M. Flaud, R. R. Gamache, J. Harrison, J.-M. Hartmann, C. Hill, J. T. Hodges, D. Jacquemart, A. Jolly, J. Lamouroux, R. J. LeRoy, G. Li, D. Long, C. J. Mackie, S. T. Massie, S. Mikhailenko, H. S. P. Müller, O. V. Naumenko, A. V. Nikitin, J. Orphal, V. I. Perevalov, A. Perrin, E. R. Polovtseva, C. Richard, M. A. H. Smith, E. Starikova, K. Sung, S. A. Tashkun, J. Tennyson, G. C. Toon, V. Tyuterev, and G. Wagner, Journal of Quantitative Spectroscopy and Radiative Transfer 130, 4 (2013).

${ }^{8}$ R. N. Zare, Angular Momentum: Understanding Spatial Aspects in Chemistry and Physics (Wiley, 1988).

${ }^{9}$ M. Abramowitz and I. A. Stegun, Handbook of Mathematical Functions with Formulas, Graphs, and Mathematical Tables, 10th ed., Applied Mathematics No. 55 (United States Department of Commerce, National Bureau of Standards, 1972).

${ }^{10}$ Stanford Research Systems, Model SR830 DSP Lock-in Amplifier User Manual (2011).

${ }^{11}$ This can be inferred via the Wiener-Khinchin theorem. The signal's autocorrelation function is the Fourier transform of the PSD of the signal. If, for simplicity's sake, we assume the PSD to be rectangular with a width of $780 \mathrm{~Hz}$, then the autocorrelation is sinc-shaped with the first zero at $\tau=(780 \mathrm{~Hz})^{-1}=1.28 \mathrm{~ms}$. Hence, two points separated by only $\delta t=10 \mu \mathrm{s}$ are strongly correlated.

${ }^{12}$ C. Y. Chang and J. T. Shy, Applied Optics 54, 8526 (2015). 